\title{
A First-in-class, Highly Selective and Cell-active Allosteric Inhibitor of Protein Arginine Methyltransferase 6 (PRMT6)
}

Yudao Shen,${ }^{\mathrm{a},+}$ Fengling Li ${ }^{\mathrm{b},+}$ Magdalena M. Szewczyk, ${ }^{\mathrm{b},+}$ Levon Halabelian, ${ }^{\mathrm{b},+}$ Irene Chau, ${ }^{\mathrm{b}}$ Mohammad S. Eram, ${ }^{\mathrm{b}}$ Carlo Dela Seña, ${ }^{\mathrm{b}}$ Kwang-Su Park, ${ }^{\mathrm{a}}$ Fanye Meng, ${ }^{a}$ He Chen, ${ }^{\mathrm{a}}$ Hong Zeng, David McLeod, ${ }^{\mathrm{e}}$ Carlos A. Zepeda-Velázquez, ${ }^{\mathrm{e}}$ Robert M. Campbell, ${ }^{\mathrm{f}}$ Mary M. Mader, ${ }^{\mathrm{f}}$ Brian M. Watson, ${ }^{\mathrm{f}}$ Matthieu Schapira, ${ }^{\mathrm{b}}$ Cheryl H. Arrowsmith, ${ }^{\mathrm{b}, \mathrm{d}}$ Rima Al-Awar, ${ }^{\mathrm{e}}$ Dalia Barsyte-Lovejoy, ${ }^{\mathrm{b}}$ H. Ümit Kaniskan, ${ }^{a}$ Peter J. Brown, ${ }^{\mathrm{b}, *}$ Masoud Vedadi, ${ }^{\mathrm{b}, \mathrm{c}, *}$ and Jian Jin ${ }^{\mathrm{a}, *}$

${ }^{a}$ Mount Sinai Center for Therapeutics Discovery, Departments of Pharmacological Sciences and Oncological Sciences, Tisch Cancer Institute, Icahn School of Medicine at Mount Sinai, New York, NY 10029, United of States

${ }^{b}$ Structural Genomics Consortium, University of Toronto, Toronto, ON M5G 1L7, Canada ${ }^{\mathrm{c}}$ Department of Pharmacology and Toxicology, University of Toronto, Toronto, ON M5S 1A8, Canada

${ }^{\mathrm{d} D e p a r t m e n t ~ o f ~ M e d i c a l ~ B i o p h y s i c s, ~ U n i v e r s i t y ~ o f ~ T o r o n t o, ~ T o r o n t o, ~ O N ~ M 5 G ~ 1 L 7, ~ C a n a d a ~}$ ${ }^{\text {e} O n t a r i o ~ I n s t i t u t e ~ f o r ~ C a n c e r ~ R e s e a r c h, ~ T o r o n t o, ~ O N ~ M 5 G ~ 0 A 3, ~ C a n a d a ~}$

fEli Lilly and Company, Lilly Research Laboratories, Indianapolis, IN 46225, United of States

\section{Supporting Information}

Table S1. Selectivity of (R)-2 and (S)-2 against methyltransferases.

Table S2. Selectivity of $(R)$-2 against non-epigenetic targets.

Table S3. Effect of preincubation on PRMT6 inhibitory potency of (R)-2.

Table S4. Crystallography data and refinement statistics. 
Figure S1. Mass spectrometry results of PRMT6 incubated with $(R)$-2.

Figure S2. Crystal structures of PRMT6 in complex with an allosteric inhibitor. 8

Figure S3. ${ }^{1} \mathrm{H}$ NMR spectrum of compound 3.

Figure S4. ${ }^{13} \mathrm{C}$ NMR spectrum of compound $3 . \quad 9$

$\begin{array}{ll}\text { Figure S5. }{ }^{1} \mathrm{H} \text { NMR spectrum of }(R)-2 . & 10\end{array}$

Figure S6. ${ }^{13} \mathrm{C}$ NMR spectrum of $(R)-\mathbf{2} .10$

Figure S7. HPLC spectrum of compound $(R)-\mathbf{2} .11$

Figure S8. ${ }^{1} \mathrm{H}$ NMR spectrum of $(S)$-2. 12

$\begin{array}{lr}\text { Figure S9. }{ }^{13} \mathrm{C} \text { NMR spectrum of }(S) \text {-2. } & 12\end{array}$

$\begin{array}{lr}\text { Figure S10. HPLC spectrum of compound (S)-2. } & 13\end{array}$ 
Table S1. Selectivity of (R)-2 (SGC6870) and (S)-2 (SGC6870N) against methyltransferases.

\begin{tabular}{|c|c|c|c|c|c|c|c|c|c|c|c|c|}
\hline \multirow{4}{*}{ Protein } & \multicolumn{12}{|c|}{ Activity\% } \\
\hline & \multicolumn{6}{|c|}{ SGC6870 } & \multicolumn{6}{|c|}{ SGC6870N } \\
\hline & \multicolumn{3}{|c|}{$1 \mu \mathrm{M}$} & \multicolumn{3}{|c|}{$10 \mu \mathrm{M}$} & \multicolumn{3}{|c|}{$1 \mu \mathrm{M}$} & \multicolumn{3}{|c|}{$10 \mu \mathrm{M}$} \\
\hline & exp1 & $\exp 2$ & $\exp 3$ & $\exp 1$ & $\exp 2$ & exp3 & $\exp 1$ & $\exp 2$ & $\exp 3$ & $\exp 1$ & $\exp 2$ & exp3 \\
\hline PRMT6 & 16 & 17 & 17 & 8 & 7 & 7 & 90 & 97 & 90 & 93 & 91 & 94 \\
\hline PRMT1 & 103 & 98 & 94 & 102 & 91 & 90 & 105 & 97 & 94 & 92 & 110 & 100 \\
\hline PRMT3 & 101 & 102 & 98 & 102 & 95 & 97 & 102 & 102 & 101 & 102 & 100 & 104 \\
\hline PRMT4 & 100 & 101 & 93 & 90 & 106 & 93 & 94 & 106 & 105 & 93 & 105 & 92 \\
\hline PRMT5 & 101 & 98 & 107 & 98 & 103 & 99 & 99 & 94 & 103 & 102 & 95 & 100 \\
\hline PRMT7 & 99 & 99 & 95 & 106 & 107 & 96 & 102 & 93 & 98 & 101 & 102 & 98 \\
\hline PRMT8 & 102 & 98 & 104 & 97 & 96 & 95 & 97 & 105 & 98 & 95 & 96 & 99 \\
\hline PRMT9 & 107 & 100 & 100 & 102 & 106 & 100 & 107 & 108 & 99 & 107 & 103 & 105 \\
\hline G9a & 91 & 92 & 105 & 105 & 91 & 93 & 102 & 96 & 89 & 101 & 98 & 96 \\
\hline GLP & 101 & 100 & 96 & 104 & 94 & 99 & 100 & 98 & 96 & 99 & 99 & 105 \\
\hline SETDB1 & 98 & 105 & 104 & 108 & 100 & 104 & 110 & 107 & 93 & 109 & 102 & 102 \\
\hline SUV39H1 & 101 & 95 & 94 & 100 & 90 & 84 & 109 & 96 & 95 & 93 & 103 & 94 \\
\hline SUV39H2 & 101 & 97 & 100 & 98 & 97 & 100 & 99 & 102 & 98 & 100 & 99 & 95 \\
\hline SUV420H1 & 105 & 102 & 101 & 106 & 96 & 100 & 96 & 106 & 107 & 103 & 105 & 101 \\
\hline SUV42OH2 & 99 & 105 & 103 & 109 & 98 & 99 & 100 & 103 & 101 & 101 & 102 & 102 \\
\hline SETD7 & 97 & 102 & 87 & 102 & 98 & 87 & 101 & 103 & 98 & 106 & 99 & 92 \\
\hline SETD8 & 104 & 100 & 99 & 104 & 93 & 79 & 100 & 100 & 94 & 100 & 103 & 104 \\
\hline MLL1 & 95 & 105 & 99 & 112 & 97 & 99 & 98 & 106 & 94 & 105 & 93 & 102 \\
\hline MLL3 & 102 & 103 & 107 & 95 & 90 & 106 & 98 & 103 & 103 & 88 & 95 & 93 \\
\hline PRDM9 & 103 & 101 & 103 & 94 & 96 & 101 & 100 & 97 & 99 & 98 & 96 & 95 \\
\hline PRC2 & 96 & 102 & 105 & 109 & 104 & 89 & 105 & 102 & 98 & 97 & 97 & 98 \\
\hline SETD2 & 99 & 107 & 105 & 107 & 96 & 99 & 103 & 94 & 97 & 91 & 104 & 100 \\
\hline SMYD2 & 102 & 100 & 101 & 100 & 96 & 94 & 98 & 94 & 91 & 92 & 93 & 98 \\
\hline SMYD3 & 104 & 107 & 90 & 105 & 95 & 95 & 97 & 96 & 99 & 101 & 108 & 96 \\
\hline BCDIN3D & 101 & 106 & 93 & 101 & 103 & 100 & 89 & 100 & 99 & 98 & 99 & 101 \\
\hline DNMT1 & 99 & 99 & 97 & 107 & 93 & 93 & 100 & 99 & 97 & 101 & 102 & 109 \\
\hline DNMT3A/3L & 99 & 100 & 98 & 111 & 92 & 94 & 104 & 99 & 92 & 90 & 87 & 102 \\
\hline DNMT3B/3L & 108 & 91 & 111 & 109 & 83 & 103 & 102 & 98 & 92 & 97 & 94 & 115 \\
\hline DOT1L & 109 & 95 & 103 & 91 & 100 & 87 & 111 & 91 & 98 & 93 & 93 & 97 \\
\hline ASH1L & 99 & 92 & 101 & 112 & 93 & 110 & 104 & 108 & 94 & 97 & 95 & 107 \\
\hline NSD1 & 102 & 93 & 96 & 106 & 96 & 107 & 106 & 102 & 93 & 99 & 94 & 112 \\
\hline NSD2 & 97 & 104 & 90 & 110 & 96 & 109 & 104 & 94 & 95 & 100 & 95 & 116 \\
\hline NSD3 & 101 & 98 & 99 & 104 & 93 & 114 & 114 & 95 & 102 & 92 & 99 & 106 \\
\hline
\end{tabular}


Table S2. Selectivity of (R)-2 against non-epigenetic targets.

\begin{tabular}{|c|c|c|}
\hline Target & $\begin{array}{l}\text { Percentage of Inhibition at } \\
\qquad 1 \mu \mathrm{M}(\%)\end{array}$ & Positive Control \\
\hline A2A (h) (agonist radioligand) & 2 & NECA \\
\hline Alpha 1A (h) (antagonist radioligand) & 4 & WB4101 \\
\hline Alpha 2A (h) (antagonist radioligand) & -8 & yohimbine \\
\hline Beta $1(\mathrm{~h})$ (agonist radioligand) & -13 & atenolol \\
\hline Beta 2 (h) (antagonist radioligand) & -7 & ICI118551 \\
\hline BZD (central) (agonist radioligand) & -9 & diazepam \\
\hline CB1 (h) (agonist radioligand) & -20 & CP55940 \\
\hline CB2 (h) (agonist radioligand) & -19 & WIN 55212-2 \\
\hline CCK1 (CCKA) (h) (agonist radioligand) & 1 & CCK-8s \\
\hline D1 (h) (antagonist radioligand) & 9 & SCH23390 \\
\hline D2S (h) (agonist radioligand) & -1 & 7-OH-DPAT \\
\hline ETA (h) (agonist radioligand) & -24 & endothelin-1 \\
\hline NMDA (antagonist radioligand) & 1 & CGS19755 \\
\hline H1 (h) (antagonist radioligand) & 0 & pyrilamine \\
\hline $\mathrm{H} 2 \mathrm{(h)}$ (antagonist radioligand) & -18 & cimetidine \\
\hline MAO-A (antagonist radioligand) & 12 & clorgyline \\
\hline M1 (h) (antagonist radioligand) & -2 & pirenzepine \\
\hline M2 (h) (antagonist radioligand) & -5 & methoctramine \\
\hline M3 (h) (antagonist radioligand) & 12 & 4-DAMP \\
\hline $\mathrm{N}$ neuronal alpha 4beta 2 (h) (agonist radioligand) & -18 & nicotine \\
\hline Delta (DOP) (h) (agonist radioligand) & -20 & DPDPE \\
\hline Kappa (h) (KOP) (agonist radioligand) & 20 & U50488 \\
\hline $\mathrm{Mu}(\mathrm{MOP})(\mathrm{h})$ (agonist radioligand) & 4 & DAMGO \\
\hline 5-HT1A (h) (agonist radioligand) & 5 & 8-OH-DPAT \\
\hline 5-HT1B (h) (antagonist radioligand) & 6 & serotonine \\
\hline 5-HT2A (h) (agonist radioligand) & 12 & $( \pm)$-DOI \\
\hline 5-HT2B (h) (agonist radioligand) & 1 & $( \pm)$-DOI \\
\hline 5-HT3 (h) (antagonist radioligand) & -5 & MDL72222 \\
\hline GR (h) (agonist radioligand) & 15 & dexamethasone \\
\hline AR (h) (agonist radioligand) & -7 & testosterone \\
\hline V1a (h) (agonist radioligand) & -5 & $\begin{array}{l}{[\mathrm{d}(\mathrm{CH} 2) 51, \mathrm{Tyr}(\mathrm{Me}) 2]-} \\
\text { AVP }\end{array}$ \\
\hline $\mathrm{Ca} 2+$ channel (L, dihydropyridine site) (antagonist radioligand) & -8 & nitrendipine \\
\hline Potassium Channel hERG (human)- [3H] Dofetilide & 0 & terfenadine \\
\hline KV channel (antagonist radioligand) & -10 & alpha-dendrotoxin \\
\hline $\mathrm{Na}+$ channel (site 2) (antagonist radioligand) & -22 & veratridine \\
\hline Norepinephrine transporter (h) (antagonist radioligand) & -4 & protriptyline \\
\hline Dopamine transporter (h) (antagonist radioligand) & 6 & BTCP \\
\hline 5-HT transporter (h) (antagonist radioligand) & -17 & imipramine \\
\hline COX1(h) & 17 & diclofenac \\
\hline COX2(h) & 8 & NS398 \\
\hline PDE3A (h) & -11 & milrinone \\
\hline PDE4D2 (h) & -12 & Ro20-1724 \\
\hline Lck kinase $(\mathrm{h})$ & 1 & staurosporine \\
\hline Acetylcholinesterase (h) & 7 & galanthamine \\
\hline
\end{tabular}

Results are from two duplicate experiments. 
Table S3. Effect of preincubation on PRMT6 inhibitory potency of (R)-2 (SGC6870).

\begin{tabular}{|c|c|c|}
\hline \multirow{2}{*}{ Preincubation (min) } & \multicolumn{2}{|c|}{ PRMT6 + SGC6870 } \\
\cline { 2 - 3 } & $\mathrm{IC}_{50}(\mu \mathrm{M})$ & Hill Slope \\
\hline 0 & $7.4 \pm 0.8$ & 0.7 \\
\hline 15 & $0.44 \pm 0.03$ & 1.2 \\
\hline 30 & $0.33 \pm 0.07$ & 1.1 \\
\hline 60 & $0.18 \pm 0.02$ & 1.5 \\
\hline 120 & $0.06 \pm 0.002$ & 1.7 \\
\hline
\end{tabular}


Table S4. Crystallography data and refinement statistics.

\begin{tabular}{|c|c|c|}
\hline & PRMT6 + (R)-1 & PRMT6 + SGC6870 \\
\hline $\begin{array}{l}\text { PDB Code } \\
\text { Data collection }\end{array}$ & Data collection & 6W6D \\
\hline $\begin{array}{l}\text { Space group } \\
\text { Cell dimensions }\end{array}$ & $14_{1}$ & $14_{1}$ \\
\hline $\begin{array}{l}a, b, c(\AA) \\
\alpha, \beta, \gamma\left(^{\circ}\right)\end{array}$ & $\begin{array}{l}94.7,94.7,108.7 \\
90.00,90.00,90.00\end{array}$ & $\begin{array}{l}94.6,94.6,108.1 \\
90.00,90.00,90.00\end{array}$ \\
\hline $\begin{array}{l}\text { Resolution }(\AA) \text { (highest } \\
\text { resolution shell) } \\
\text { Measured reflections }\end{array}$ & $50.00-1.98(2.01-1.98)$ & $50.00-1.91(1.94-1.91)$ \\
\hline Unique reflections & 33067 & 36823 \\
\hline $\begin{array}{l}R_{\text {merge }} \\
I / \sigma l \\
\text { Completeness (\%) } \\
\text { Redundancy }\end{array}$ & $\begin{array}{l}6.0(90.7) \\
47.0(2.3) \\
100.0(100.0) \\
7.7(7.7)\end{array}$ & $\begin{array}{l}7.6(97.6) \\
35.4(1.7) \\
99.8(99.9) \\
6.2(6.1)\end{array}$ \\
\hline \multicolumn{3}{|l|}{ Refinement } \\
\hline Resolution $(\AA)$ & $50.00-1.98$ & $47.35-1.91$ \\
\hline No. reflections (test set) & 32078(966) & $35321(1487)$ \\
\hline$R_{\text {work } /} R_{\text {free }}(\%)$ & $20.0 / 20.8$ & $18.1 / 20.9$ \\
\hline \multicolumn{3}{|l|}{ No. atoms } \\
\hline Protein & 2604 & 2563 \\
\hline Co-factor & 26 & 26 \\
\hline Compound & 28 & 29 \\
\hline Water & 102 & 132 \\
\hline \multicolumn{3}{|l|}{ B-factors $\left(\AA^{2}\right)$} \\
\hline Protein & 46.4 & 40.9 \\
\hline Co-factor & 49.8 & 39.4 \\
\hline Compound & 40.8 & 32.0 \\
\hline Water & 48.1 & 43.9 \\
\hline \multicolumn{3}{|l|}{ RMSD } \\
\hline Bond lengths $(\AA ̊)$ & 0.009 & 0.008 \\
\hline Bond angles $\left({ }^{\circ}\right)$ & 1.372 & 1.446 \\
\hline \multicolumn{3}{|l|}{$\begin{array}{l}\text { Ramachandran plot \% } \\
\text { residues }\end{array}$} \\
\hline Favored & 97.7 & 96.7 \\
\hline Additional allowed & 2.3 & 3.3 \\
\hline Generously allowed & 0.0 & 0.0 \\
\hline Disallowed & 0.0 & 0.0 \\
\hline
\end{tabular}



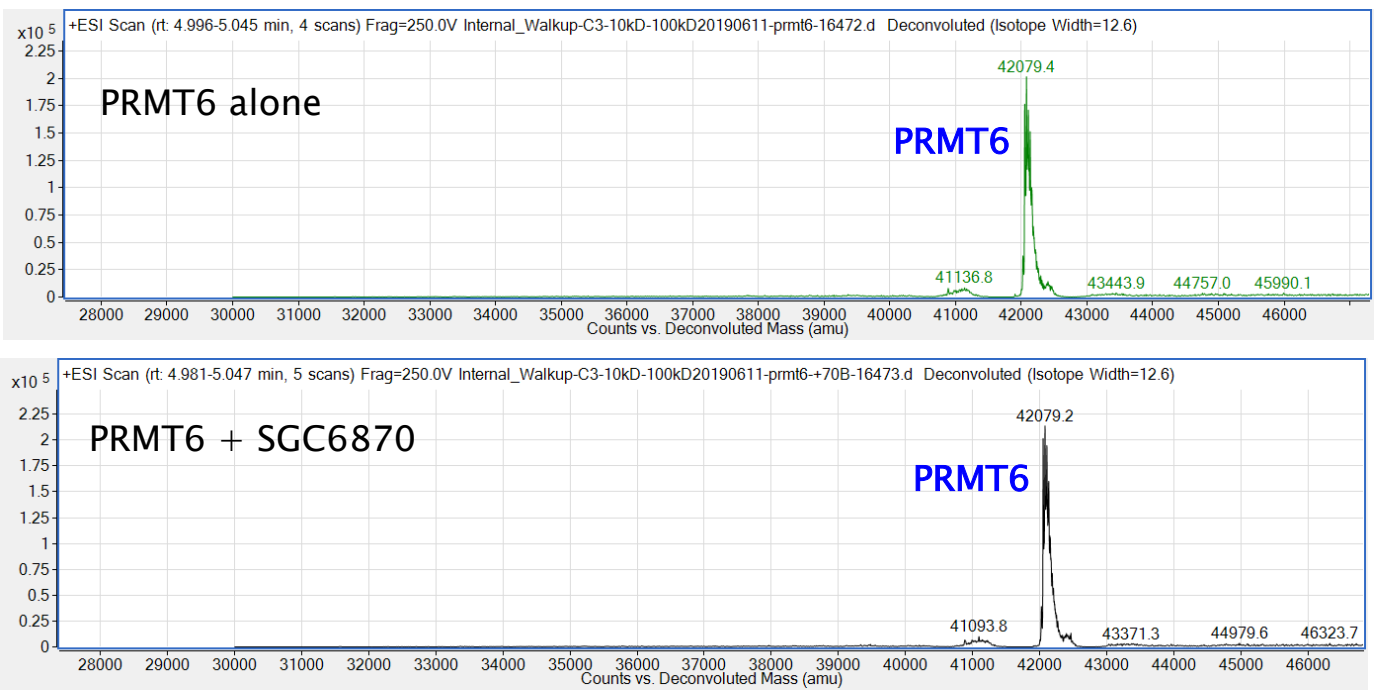

Figure S1. Mass spectrometry results of PRMT6 incubated with (R)-2 (SGC6870) indicate that (R)-2 did not covalently modify PRMT6. 
A

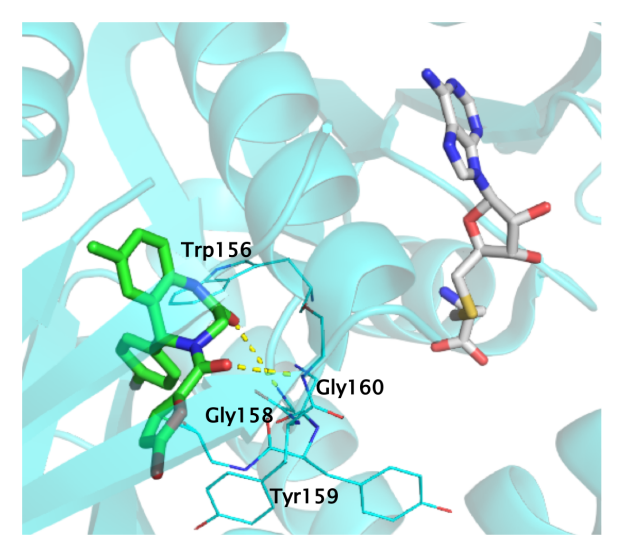

B

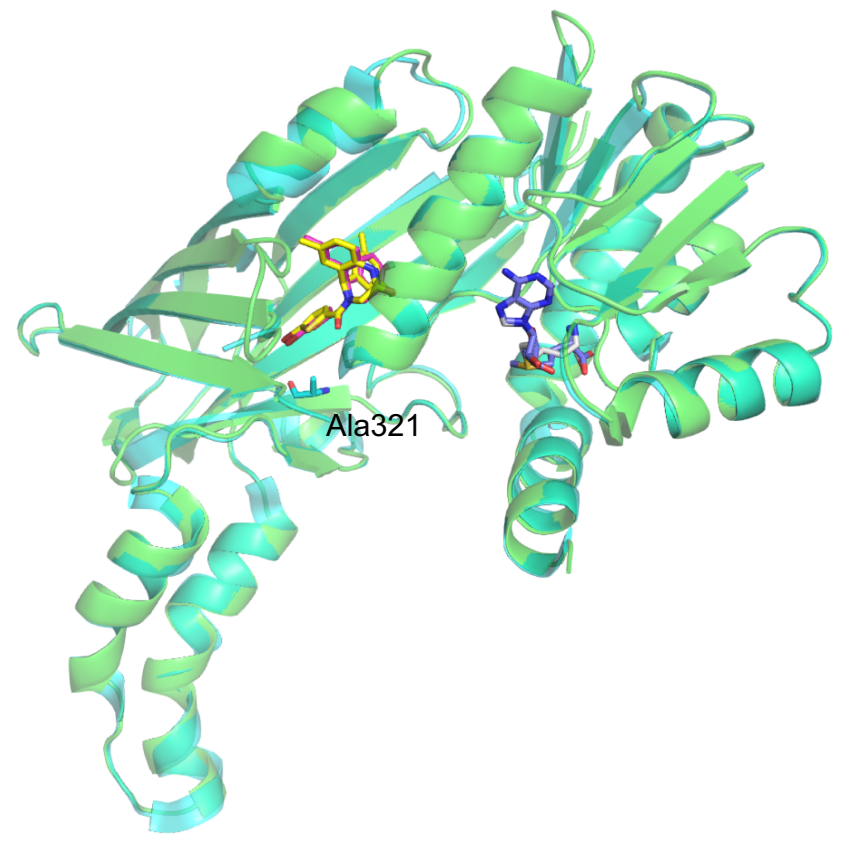

C

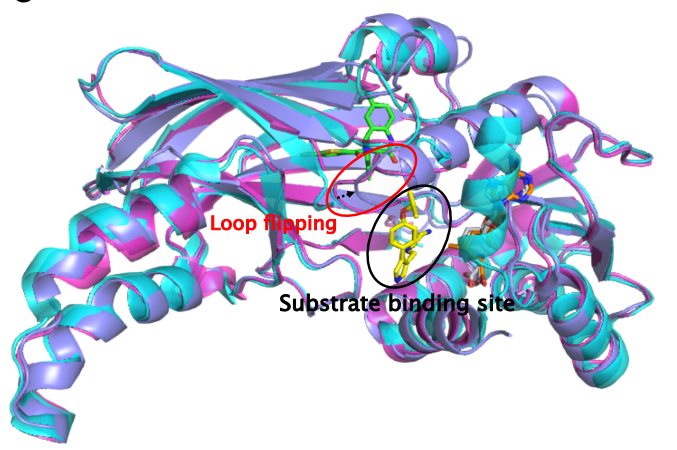

Figure S2. Crystal structures of PRMT6 in complex with an allosteric inhibitor. (A) Crystal structure of PRMT6 (cyan) in complex with (R)-1 (green), SAH (gray), (PDB: 5WCF). Key hydrogen bond interactions are highlighted in yellow dotted lines. (B) Structural alignment of PRMT6 (cyan)-(R)-1 (magenta)-SAH (gray) and PRMT6 (green)- (R)-2 (yellow)-SAM (blue). (C) Structural alignments of complexes PRMT6 (tint)-(R)-2 (green)-SAM (orange), PRMT6 (cyan)-MS023 (yellow)-SAH (gray) (PDB: 5E8R) and PRMT6 (magenta)-SAH (gray) (PDB: $4 \mathrm{C} 05)$. 


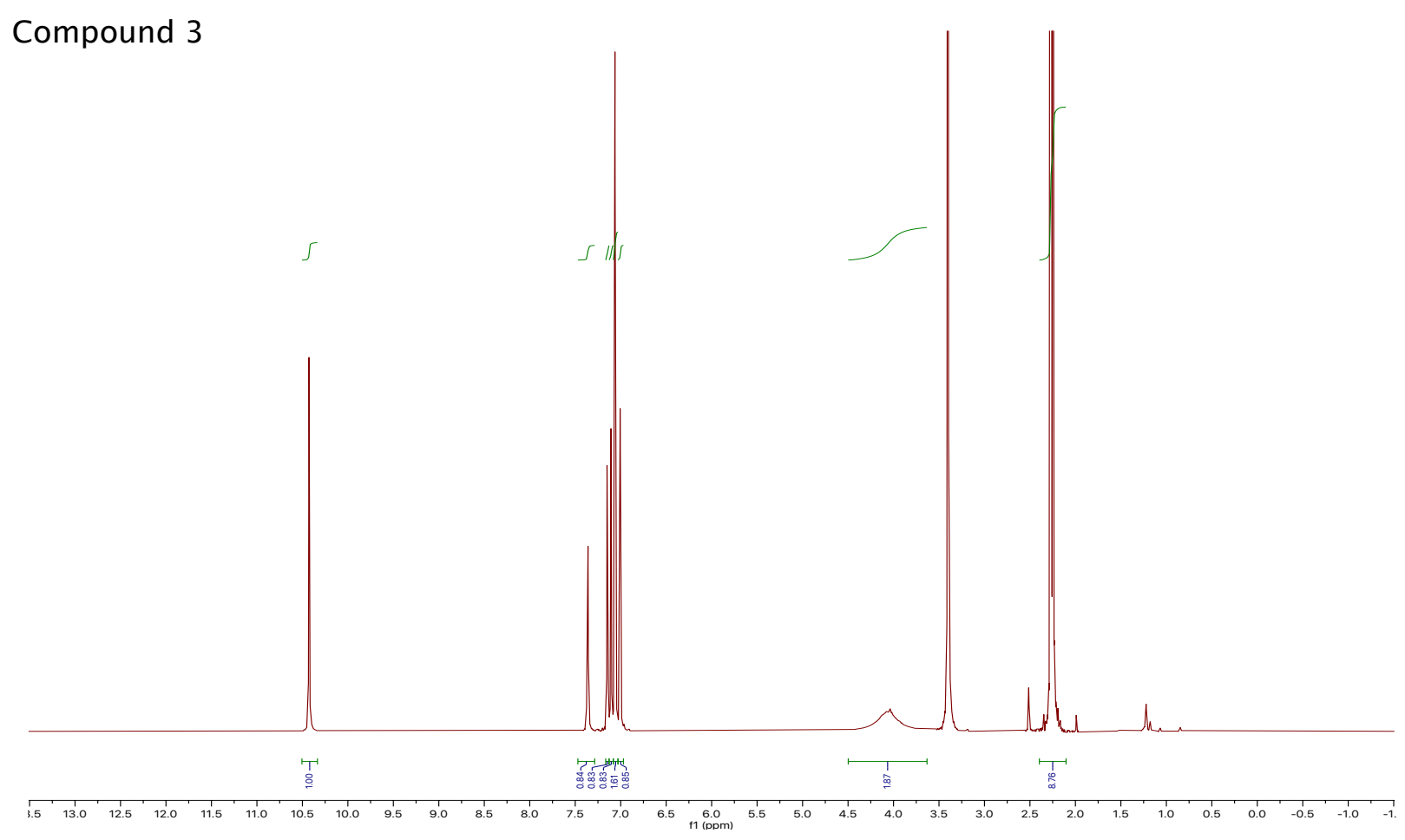

Figure S3. ${ }^{1}$ H NMR spectrum of compound 3.
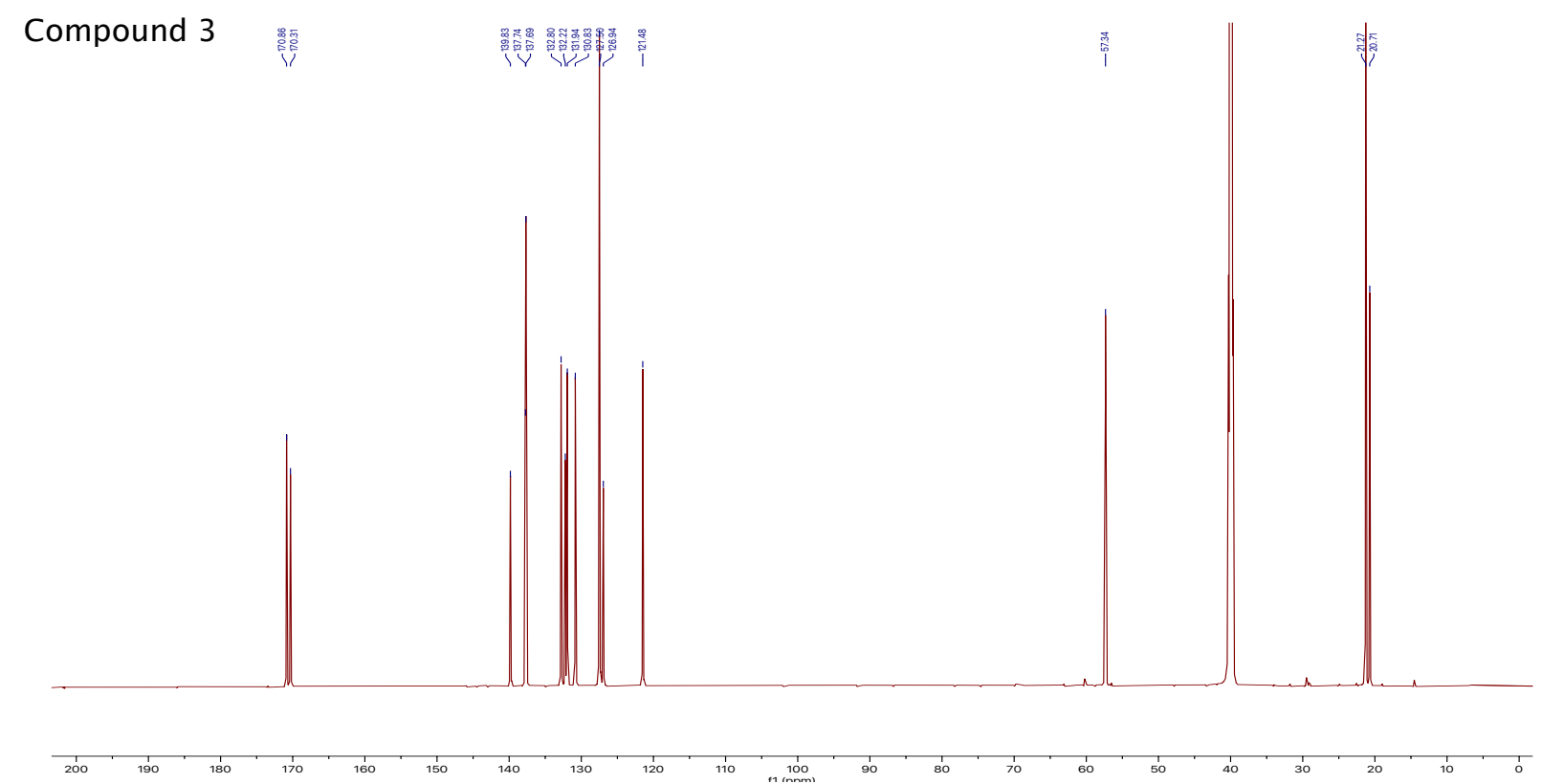

Figure S4. ${ }^{13} \mathrm{C}$ NMR spectrum of compound 3. 


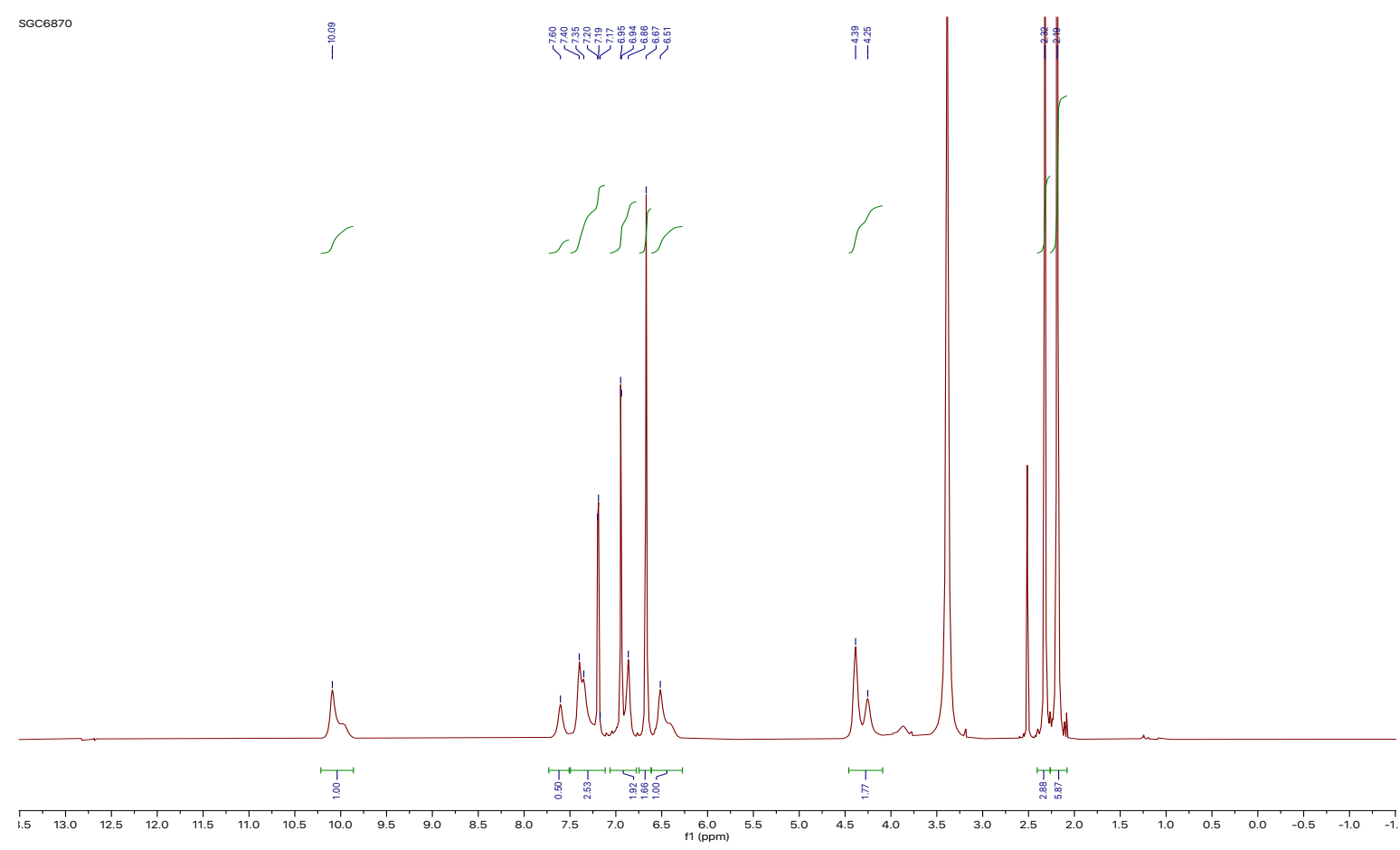

Figure S5. ${ }^{1} \mathrm{H}$ NMR spectrum of compound (R)-2.
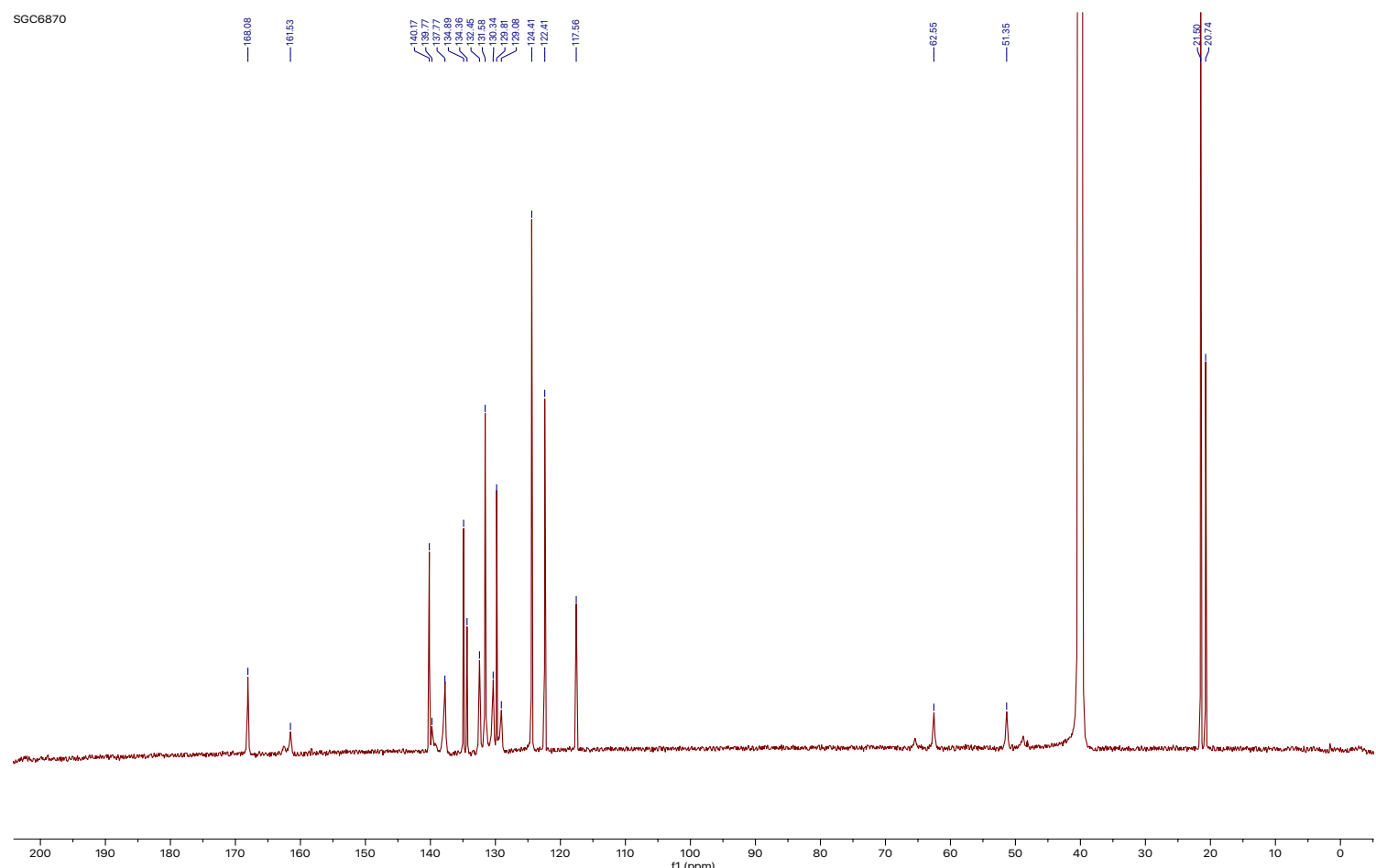

Figure S6. ${ }^{13} \mathrm{C}$ NMR spectrum of compound $(R)-2$. 


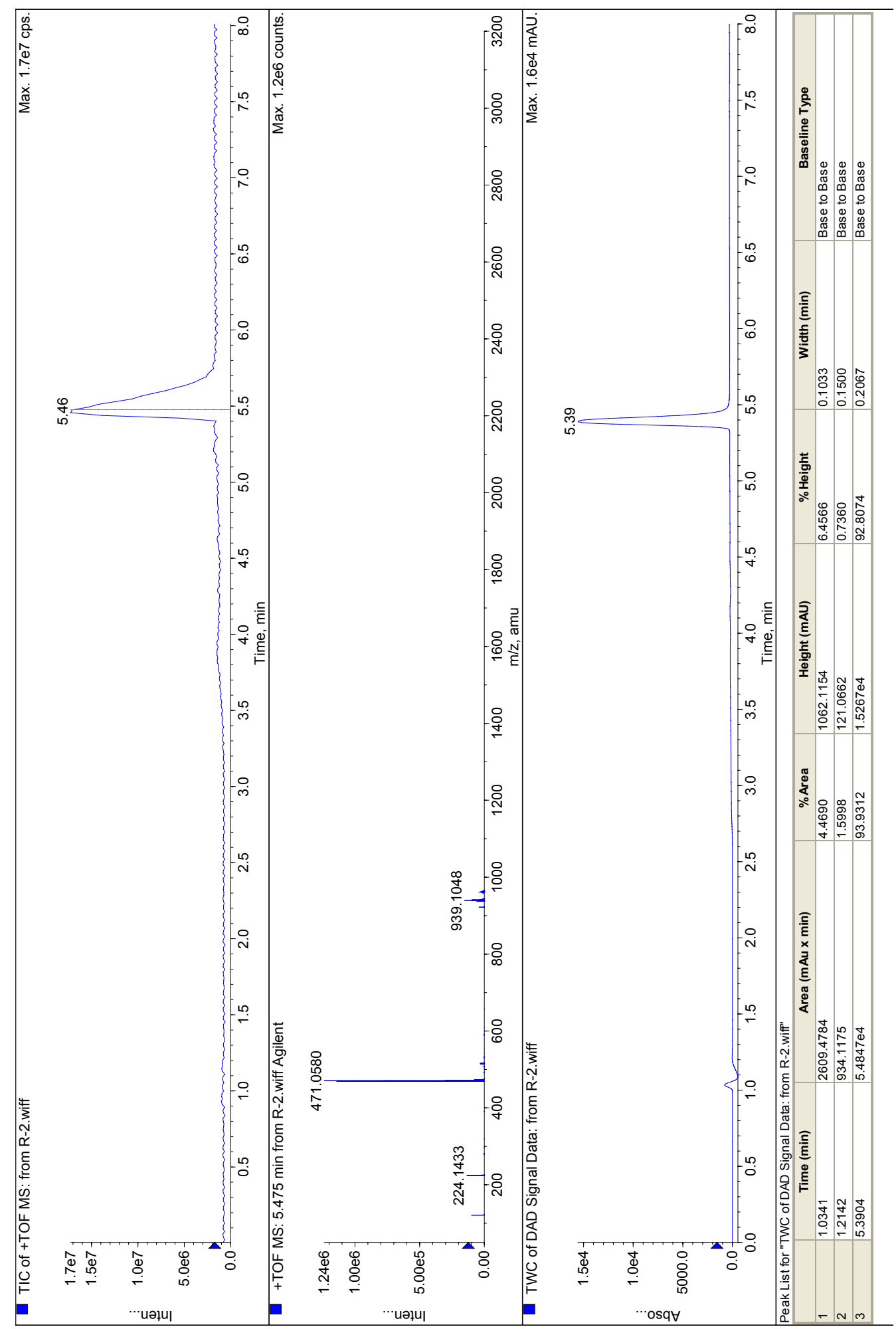

Figure S7. HPLC spectrum of compound $(R)-2$. Solvent peaks are detected at $1 \min \left(t_{\mathrm{R}}\right)$ and 1.2 $\min \left(t_{\mathrm{R}}\right)$. Purity of $(R)-2$ is $>95 \%$. 


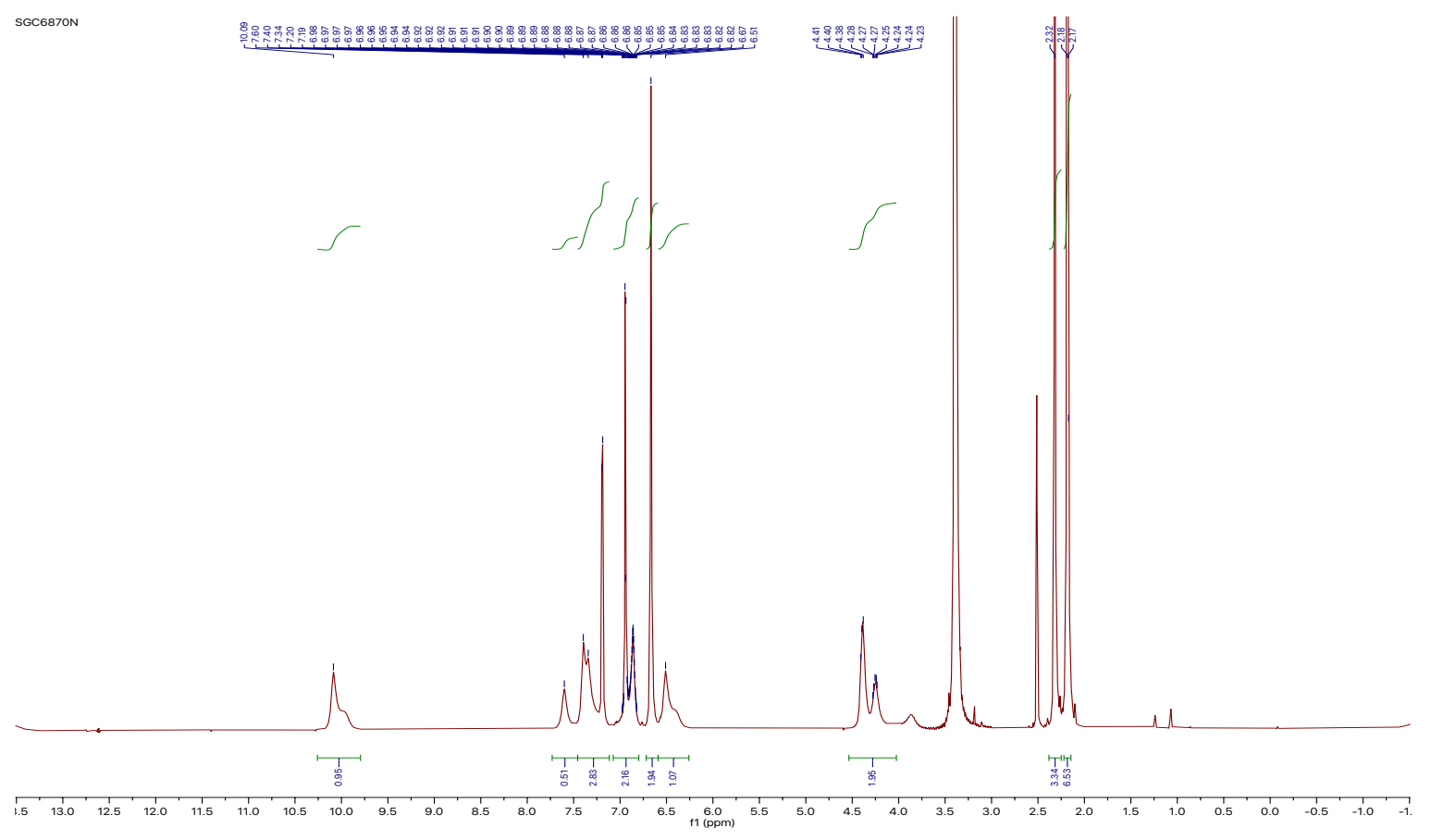

Figure S8. ${ }^{1} \mathrm{H}$ NMR spectrum of compound (S)-2.

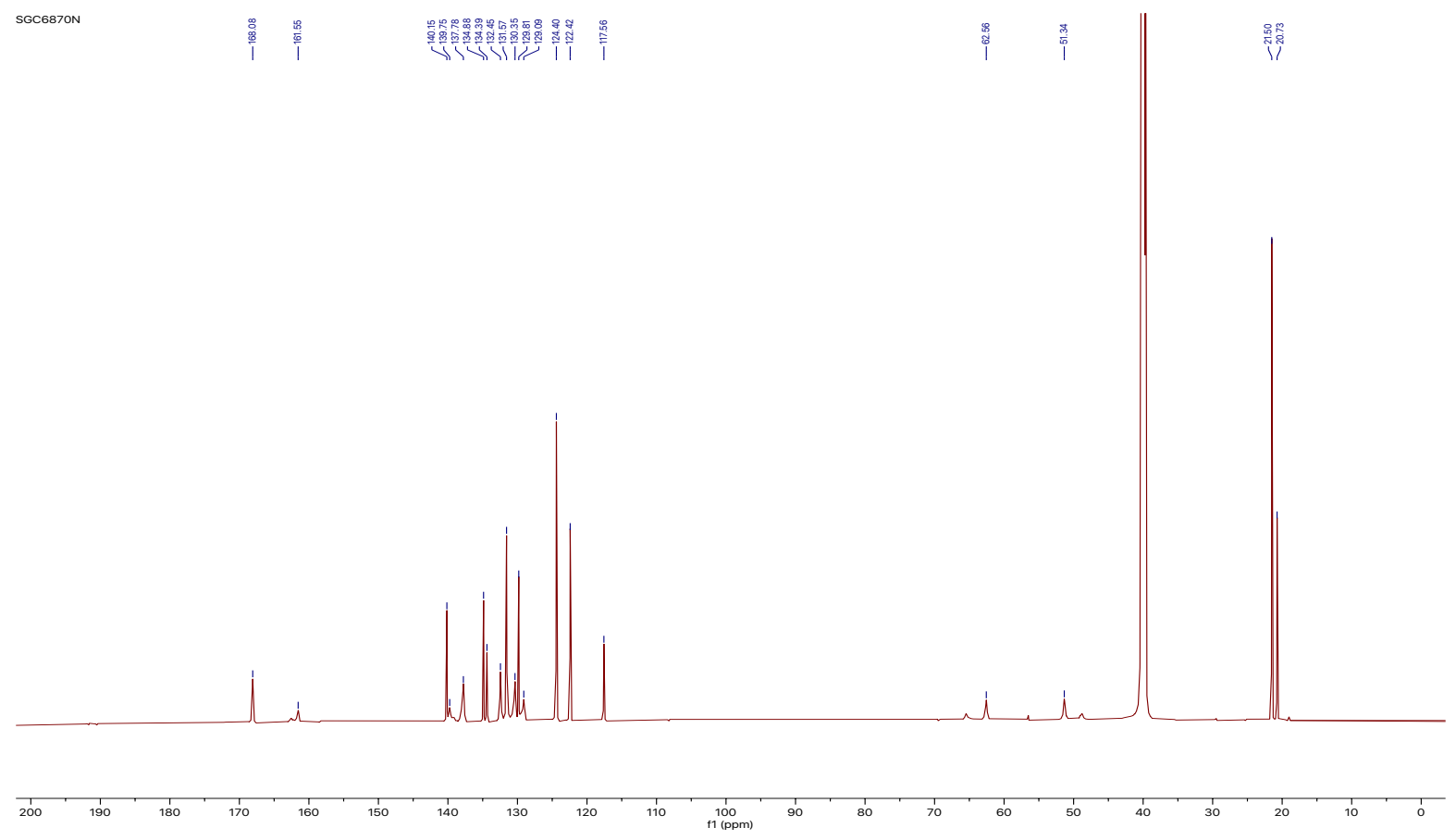

Figure S9. ${ }^{13} \mathrm{C}$ NMR spectrum of compound $(S)$-2. 


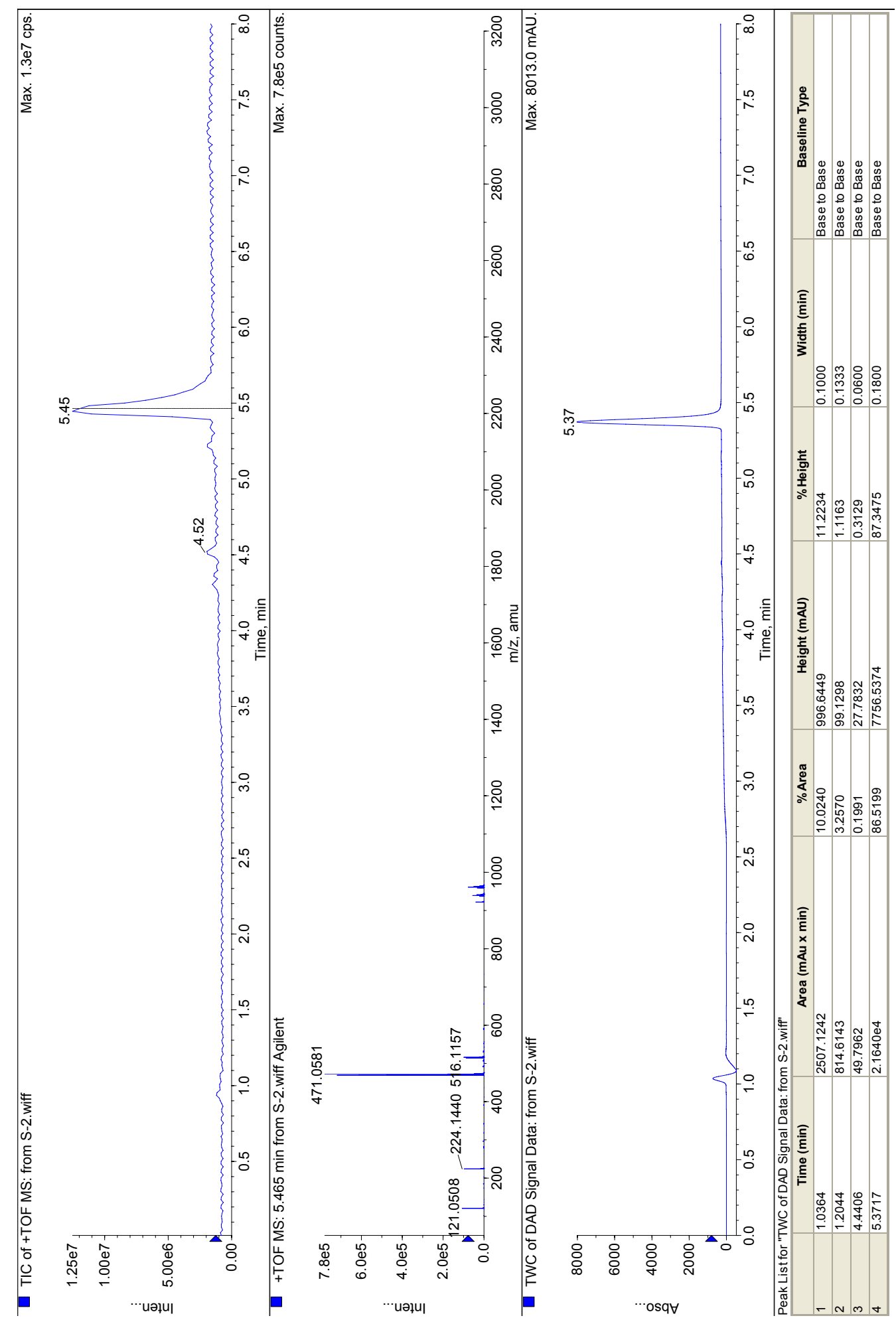

Figure S10. HPLC spectrum of compound $(S)-2$. Solvent peaks are detected at $1 \mathrm{~min}\left(t_{\mathrm{R}}\right)$ and 1.2 $\min \left(t_{\mathrm{R}}\right)$. Purity of $(S)-2$ is $>95 \%$. 